\title{
Polyanalytic boundary value problems for planar domains with harmonic Green function
}

\author{
Heinrich Begehr ${ }^{1}\left[\right.$ [D $\cdot$ Bibinur Shupeyeva ${ }^{2}$
}

Received: 22 December 2020 / Revised: 16 June 2021 / Accepted: 21 June 2021

/ Published online: 7 July 2021

(c) The Author(s) 2021

\begin{abstract}
There are three basic boundary value problems for the inhomogeneous polyanalytic equation in planar domains, the well-posed iterated Schwarz problem, and further two over-determined iterated problems of Dirichlet and Neumann type. These problems are investigated in planar domains having a harmonic Green function. For the Schwarz problem, treated earlier [Ü. Aksoy, H. Begehr, A.O. Çelebi, AV Bitsadze's observation on bianalytic functions and the Schwarz problem. Complex Var Elliptic Equ 64(8): 1257-1274 (2019)], just a modification is mentioned. While the Dirichlet problem is completely discussed for arbitrary order, the Neumann problem is just handled for order up to three. But a generalization to arbitrary order is likely.
\end{abstract}

Keywords Poly-analytic · Cauchy-Schwarz-Pompeiu representation · Green function · Schwarz - Dirichlet - Neumann boundary value problems - Admissible domain $\cdot$ Ring domain $\cdot \mathrm{Bi}$ - and tri-analytic Pompeiu integral operators

Mathematics Subject Classification 30E25 $\cdot 30 \mathrm{G} 20 \cdot 31 \mathrm{~A} 10 \cdot 31 \mathrm{~A} 25 \cdot 35 \mathrm{~J} 40$

\section{Introduction}

In the endeavor to build a general theory for complex partial differential equations the study of model equations is a further step after extensive investigations of basic

Dedicated to Professor S.A. Abdymanapov on the occasion of his 70th birthday.

$\bowtie$ Heinrich Begehr

begehrh@zedat.fu-berlin.de

Bibinur Shupeyeva

bibinur.shupeyeva@nu.edu.kz

1 Mathematisches Institut, FU Berlin, Arnimallee 3, 14195 Berlin, Germany

2 Nazarbayev University, 53 Kabanbay Batyr Ave, 010000 Nur-Sultan, Kazakhstan

Birkhäuser 
equations as the Cauchy-Riemann and the Poisson equation. There are two kinds of model equations, the polyanalytic and the polyharmonic equation. The present considerations are restricted to the polyanalytic equation $\partial_{\bar{z}}^{n} w=f$ for natural $n \in \mathbb{N}$. The case $n=2$ is the Bitsadze equation and is related to bianalytic functions. As the polyanalytic equation can be decomposed into a system of $n$ inhomogeneous CauchyRiemann equations $n$ boundary conditions may be imposed.

For the Cauchy-Riemann equation the natural boundary value problem is the Schwarz problem, prescribing the boundary values of the real part of the function and additionally fixing the value of its imaginary part in just one point of the domain under consideration. For particular domains as the unit disc the solution is given in explicit form by proper modification of the Cauchy-Pompeiu formula [1-3,5,11,22,23,27,30]. For certain other simply connected domains having a harmonic Green function an explicit representation formula is also available [6-8].

Two other, but ill-posed problems are the Dirichlet and the Neumann boundary value problems, prescribing on one hand the boundary values of the function and on the other those of its normal derivative together with a normalization condition. For these over-determined boundary value problems solvability conditions need to be determined. In case of the unit disc they are explicitly given [11]. For general domains they are available at least in the case that the domain is equipped with a harmonic Green function, see [6] for $n=2$.

Having these three different boundary value problems for the Cauchy-Riemann equation in mind, all possible combinations of them are candidates for posing boundary values for the polyanalytic equation, see for the Bitsadze equation [6,7], where besides the iterated Schwarz, Dirichlet and Neumann problems also the Dirichlet-Neumann and Neumann-Dirichlet problems are studied.

Further investigation will here be restricted to the iterated Schwarz, the iterated Dirichlet and the iterated Neumann boundary value problems for the inhomogeneous polyanalytic equation. The first listed problem was treated in [6] already for certain simply connected domains having a harmonic Green function. In Sect. 2 this result is slightly modified in replacing the origin by any arbitrary fixed point of the domain for the side conditions. In order to explain that the restriction to admissible simply connected domains is sufficient but not necessary for treating the Schwarz problem in domains with harmonic Green function, the concentric circular ring domain is borrowed from [28] in Sect. 3.

The polyanalytic Cauchy-Pompeiu representation formula immediately provides the solution to the Dirichlet problem for any $n \in \mathbb{N}$ in cases the solution exists, Sect. 4. The solvability conditions, guaranteeing that the Cauchy-Pompeiu formula gives the solution, are expressed through the harmonic Green function for the domain. This generalizes the result for the Bitsadze equation from [6].

Finally in Sect. 5 the Neumann problem is treated for domains with harmonic Green functions. The respective result for the Bitsadze equation from [6] is modified in order to handle the problem for the trianalytic equation. For convenience the result for the Cauchy-Riemann equation is repeated. The introduction of a proper modified Pompeiu integral operator for the cases $n=2$ and $n=3$ gives an idea how to proceed for general $n$. 


\subsection{Basics for the polyanalytic operator}

The fundamental solution to the polyanalytic operator of order $n$ is given as [17]

$$
-\frac{1}{\pi(n-1) !} \frac{\bar{z}^{n-1}}{z}
$$

Lemma 1 The polyanalytic fundamental solution satisfies for regular domains $D$

$$
\begin{aligned}
& \frac{1}{2 \pi i} \int_{\partial D} \frac{1}{n !} \frac{(\overline{\zeta-z})^{n}}{\zeta-z} d \zeta=\frac{1}{\pi} \int_{D} \frac{1}{(n-1) !} \frac{(\overline{\zeta-z})^{n-1}}{\zeta-z} d \xi d \eta, \quad n \in \mathbb{N}, \\
& \frac{1}{n !} \frac{(\overline{\zeta-z})^{n}}{\zeta-z}=\frac{1}{2 \pi i} \int_{\partial D} \frac{1}{n !} \frac{(\widetilde{\zeta}-z)^{n}}{\widetilde{\zeta}-z} \frac{d \widetilde{\zeta}}{\widetilde{\zeta}-\zeta}-\frac{1}{\pi} \int_{D} \frac{1}{(n-1) !} \frac{(\widetilde{\zeta}-z)^{n-1}}{\widetilde{\zeta}-z} \frac{d \widetilde{\xi} d \widetilde{\zeta}}{\widetilde{\zeta}-\zeta}
\end{aligned}
$$

Moreover, for any function $\varphi$, analytic in $D$,

$$
\frac{1}{2 \pi i} \int_{\partial D} \frac{1}{n !} \frac{(\overline{\zeta-z})^{n}}{\zeta-z} \varphi(\zeta) d \zeta=\frac{1}{\pi} \int_{D} \frac{1}{(n-1) !} \frac{(\overline{\zeta-z})^{n-1}}{\zeta-z} \varphi(\zeta) d \xi d \eta, \quad n \in \mathbb{N},
$$

holds.

Remark 1 In particular with the harmonic part of the harmonic Green function for $D$, $G_{1}(z, \zeta)=h_{1}(z, \zeta)-\log |\zeta-z|^{2}$,

$$
\frac{1}{2 \pi i} \int_{\partial D} \frac{1}{n !} \frac{(\overline{\zeta-z})^{n}}{\zeta-z} h_{1 \zeta}(\zeta, \widehat{z}) d \zeta=\frac{1}{\pi} \int_{D} \frac{1}{(n-1) !} \frac{(\overline{\zeta-z})^{n-1}}{\zeta-z} h_{1 \zeta}(\zeta, \widehat{z}) d \xi d \eta, \quad n \in \mathbb{N}
$$

holds for $z, \widehat{z} \in D$. Although as well this relation as the formulas in Lemma 1 will not be used later on, they seem to be of own interest.

Proof While the first two relations are just consequences from the Gauss and CauchyPompeiu theorems $[10,11]$, the last formula needs a manifestation. The Cauchy theorem implies for analytic functions $\varphi$

$$
\varphi(z)=\frac{1}{2 \pi i} \int_{\partial D} \varphi(\zeta) \frac{d \zeta}{\zeta-z}
$$

and from the Cauchy-Pompeiu representation

$$
\bar{z} \varphi(z)=\frac{1}{2 \pi i} \int_{\partial D} \bar{\zeta} \varphi(\zeta) \frac{d \zeta}{\zeta-z}-\frac{1}{\pi} \int_{D} \varphi(\zeta) \frac{d \xi d \eta}{\zeta-z}
$$


is seen. Multiplying the first formula with $\bar{z}$ and subtracting both relations, verify (1) for $n=1$. For higher powers analogously arguing the relations

$$
\frac{\bar{z}^{n-v}}{(n-v) !} \varphi(z)=\frac{1}{2 \pi i} \int_{\partial D} \frac{\bar{\zeta}^{n-v}}{(n-v) !} \varphi(\zeta) \frac{d \zeta}{\zeta-z}-\frac{1}{\pi} \int_{D} \frac{\bar{\zeta}^{n-v-1}}{(n-v-1) !} \varphi(\zeta) \frac{d \xi d \eta}{\zeta-z}
$$

multiplied with $(-1)^{v} \frac{n !}{v !} \bar{z}^{v}$ and added up for $v$ between 0 and $n$ lead to

$$
0=\frac{1}{2 \pi i} \int_{\partial D} \frac{(\overline{\zeta-z})^{n}}{\zeta-z} \varphi(\zeta) d \zeta-\frac{n}{\pi} \int_{D} \frac{(\overline{\zeta-z})^{(n-1)}}{\zeta-z} \varphi(\zeta) d \xi d \eta
$$

The basic representation formula related to the polyanalytic operator, see e.g. [18], is the

Cauchy-Pompeiu representation formula. Any function $w \in C^{n}(D ; \mathbb{C}) \cap C^{n-1}(\bar{D} ; \mathbb{C})$, where $D$ is some regular domain in $\mathbb{C}$ and $n \in \mathbb{N}$, can be represented as

$$
w(z)=\sum_{\mu=0}^{n-1} \frac{1}{2 \pi i} \int_{\partial D} \frac{(-1)^{\mu}(\overline{\zeta-z})^{\mu}}{\mu !(\zeta-z)} \partial_{\bar{\zeta}}^{\mu} w(\zeta) d \zeta-\frac{1}{\pi} \int_{D} \frac{(-1)^{n-1}(\overline{\zeta-z})^{n-1}}{(n-1) !(\zeta-z)} \partial_{\bar{\zeta}}^{n} w(\zeta) d \xi d \eta
$$

\section{Polyanalytic Schwarz problem}

As an analytic function is uniquely determined by the boundary values of its real part up to an additive imaginary constant, also polyanalytic functions are given up to polyanalytic polynomials of lower order through the boundary values of the real parts of their lower order $\bar{z}$-derivatives.

Definition (n-Schwarz problem.) Find in a domain $D$ of the complex plane $\mathbb{C}$ with a fixed $z_{0} \in D$ and $n \in \mathbb{N}$ a solution to

$$
\partial_{\bar{z}}^{n} w=f \text { in } D, \quad \operatorname{Re} \partial_{\bar{z}}^{\mu} w=\gamma_{\mu} \text { on } \partial D, \quad \operatorname{Im} \partial_{\bar{z}}^{\mu} w\left(z_{0}\right)=c_{\mu}, \quad 0 \leq \mu \leq n-1,
$$

for given data $f \in L_{p}(D ; \mathbb{C}), 2<p, \gamma_{\mu} \in C(\partial D ; ; \mathbb{R}), c_{\mu} \in \mathbb{R}$.

For solving the Schwarz problem the Cauchy-Pompeiu representation is modified. It provides the unique solution to the n-Schwarz problem. At first, see [6,33,34], the case $n=1$ is investigated.

Theorem 1 (Cauchy-Schwarz-Pompeiu representation.) Any $w \in C^{1}(D ; \mathbb{C}) \cap$ $C(\bar{D} ; \mathbb{C})$ for a simply connected admissible domain $D$ with Green function $G_{1}(z, \zeta)=$ $h_{1}(z, \zeta)-\log |\zeta-z|^{2}$, and some arbitrarily fixed point $z_{0} \in D$ can be represented as

$$
w(z)=i \operatorname{Imw}\left(z_{0}\right)+\frac{1}{2 \pi i} \int_{\partial D} \operatorname{Rew}(\zeta)\left[\frac{\zeta-z_{0}}{\zeta-z} \frac{d \zeta}{\zeta-z_{0}}+\left(h_{1 \bar{\zeta}}(z, \zeta)-\frac{1}{\overline{\zeta-z_{0}}}\right) d \bar{\zeta}\right]
$$




$$
\begin{aligned}
& -\frac{1}{2 \pi} \int_{D}\left\{w_{\bar{\zeta}}(\zeta)\left[\frac{\zeta+z-2 z_{0}}{\zeta-z} \frac{1}{\zeta-z_{0}}-h_{1 \zeta}\left(z_{0}, \zeta\right)\right]\right. \\
& \left.-\overline{w_{\bar{\zeta}}(\zeta)}\left[2 h_{1 \bar{\zeta}}(z, \zeta)-h_{1 \bar{\zeta}}\left(z_{0}, \zeta\right)-\frac{1}{\overline{\zeta-z_{0}}}\right]\right\} d \xi d \eta
\end{aligned}
$$

Remark 2 Admissible for a domain $D$ means that for any $\zeta \in \partial D$ the function $h_{1 \bar{\zeta}}(\cdot, \zeta)$ is analytic in $D$, see [6,7]. The reason for this demand is, see [6], Lemma 3, that in proving the Cauchy-Schwarz-Pompeiu representation the integral about the imaginary part of the function $w$ is required to be constant in the representation

$$
\begin{aligned}
w(z)= & \frac{1}{2 \pi i} \int_{\partial D} \operatorname{Re} w(\zeta)\left[\frac{d \zeta}{\zeta-z}+h_{1 \bar{\zeta}}(z, \zeta) d \bar{\zeta}\right]+\frac{i}{2 \pi i} \int_{\partial D} \operatorname{Im} w(\zeta)\left[\frac{d \zeta}{\zeta-z}-h_{1 \bar{\zeta}}(z, \zeta) d \bar{\zeta}\right] \\
& -\frac{1}{\pi} \int_{D}\left[w_{\bar{\zeta}}(\zeta) \frac{1}{\zeta-z}-\overline{w_{\bar{\zeta}}(\zeta)} h_{1 \bar{\zeta}}(z, \zeta)\right] d \xi d \eta
\end{aligned}
$$

A condition for admissible domains and examples are given in [7].

Helpful for operating with the Green function is the differential relation [6]

$$
\frac{d \zeta}{\zeta-z}-h_{1 \bar{\zeta}}(z ; \zeta) d \bar{\zeta}=-\left[\overline{\left.\frac{d \zeta}{\zeta-z}-h_{1 \bar{\zeta}}(z ; \zeta) d \bar{\zeta}\right]}, \zeta \in \partial D, z \in D\right.
$$

a consequence of its boundary behavior $G_{1}(z ; \zeta)=0$ for $\zeta$ on $\partial D, z \in D$. Moreover, for the outward normal derivative $\partial_{v_{\zeta}}$ on the boundary $\partial D$ with the arc length parameter $s_{\zeta}$ for any $z \in D$ the relation

$$
-i \partial_{\nu_{\zeta}} G_{1}(z ; \zeta) d s_{\zeta}=\frac{d \zeta}{\zeta-z}-\frac{d \bar{\zeta}}{\overline{\zeta-z}}-h_{1 \zeta}(z, \zeta) d \zeta+h_{1 \bar{\zeta}}(z, \zeta) d \bar{\zeta}
$$

holds.

Iterating the above representation leads to the general Cauchy-Schwarz-Pompeiu formula which coincides in the case of the unit disc $D=\mathbb{D}$ with the respective representation in [10,19].

Theorem 2 Any $w \in C^{n}(D ; \mathbb{C}) \cap C^{n-1}(\bar{D} ; \mathbb{C})$ for an admissible domain $D, z_{0} \in D$, in the complex plane $\mathbb{C}$ with Green function $G_{1}(z, \zeta)$ is representable as

$$
\begin{aligned}
& w(z)=\sum_{\mu=0}^{n-1}\left\{\frac{i \operatorname{Im} \partial_{\bar{z}}^{\mu} w\left(z_{0}\right)}{\mu !}\left(z-z_{0}+\overline{z-z_{0}}\right)^{\mu}\right. \\
& +\frac{(-1)^{\mu}}{2 \pi i \mu !} \int_{\partial D} \operatorname{Re} \partial_{\bar{\zeta}}^{\mu} w(\zeta)(\zeta-z+\overline{\zeta-z})^{\mu} \\
& \left.\times\left[\frac{\zeta-z_{0}}{(\zeta-z)\left(\zeta-z_{0}\right)} d \zeta+\left(h_{1 \bar{\zeta}}(z, \zeta)-\frac{1}{\overline{\zeta-z_{0}}}\right) d \bar{\zeta}\right]\right\} \\
& +\frac{(-1)^{n}}{2 \pi(n-1) !} \int_{D}\left\{\partial \frac{n}{\zeta} w(\zeta)\left[\frac{\zeta+z-2 z_{0}}{(\zeta-z)\left(\zeta-z_{0}\right)}-h_{1 \zeta}\left(z_{0}, \zeta\right)\right]\right.
\end{aligned}
$$




$$
\left.-\overline{\partial \frac{n}{\zeta} w(\zeta)}\left[2 h_{1 \bar{\zeta}}(z, \zeta)-h_{1 \bar{\zeta}}\left(z_{0}, \zeta\right)-\frac{1}{\overline{\zeta-z_{0}}}\right]\right\}(\zeta-z+\overline{\zeta-z})^{n-1} d \xi d \eta
$$

This formula is deduced in [6] for $z_{0}=0$.

\section{Cauchy-Schwarz-Pompeiu representation for a circular ring domain}

The representation formula (3) holds for any planar domain with a Green function. It can be used to deduce a Cauchy-Schwarz-Pompeiu representation directly although $h_{1 \bar{\zeta}}(\cdot, \zeta)$ is not analytic. This is achieved exemplarily for the doubly connected circular ring $R=\{0<r<|z|<1\}$. Its harmonic Green function is, see [28,29],

$$
G_{1}(z, \zeta)=\frac{\log |z|^{2} \log |\zeta|^{2}}{\log r^{2}}+\log \left|\frac{1-z \bar{\zeta}}{\zeta-z}\right|^{2}-\sum_{n=1}^{\infty} \log \left|\frac{\left(z-r^{2 n} \zeta\right)\left(\zeta-r^{2 n} z\right)}{\left(z \bar{\zeta}-r^{2 n}\right)\left(1-r^{2 n} z \bar{\zeta}\right)}\right|^{2}
$$

For $\zeta \in \partial R, z \in R$

$$
\frac{d \zeta}{\zeta-z} \pm h_{1 \bar{\zeta}}(z, \zeta) d \bar{\zeta}=\left[\frac{\zeta}{\zeta-z} \mp \frac{\log |z|^{2}}{\log r^{2}} \pm \frac{z \bar{\zeta}}{1-z \bar{\zeta}} \pm\left(\overline{\Sigma_{1}}+\Sigma_{2}\right)\right] \frac{d \zeta}{\zeta}
$$

with

$$
\Sigma_{1}=\sum_{n=1}^{\infty}\left(\frac{r^{2 n} \zeta}{r^{2 n} \zeta-z}+\frac{r^{2 n} z}{\zeta-r^{2 n} z}\right), \quad \Sigma_{2}=\sum_{n=1}^{\infty}\left(\frac{r^{2 n}}{r^{2 n}-z \bar{\zeta}}+\frac{r^{2 n} z \bar{\zeta}}{1-r^{2 n} z \bar{\zeta}}\right) .
$$

Obviously for $R$ the derivative $h_{1 \bar{\zeta}}(\cdot, \zeta)$ is not analytic. In particular

$$
\frac{z \bar{\zeta}}{1-z \bar{\zeta}}+\overline{\Sigma_{1}}+\Sigma_{2}=\left\{\begin{array}{l}
\frac{z}{\zeta-z}+\Sigma_{1}+\overline{\Sigma_{1}},|\zeta|=1 \\
\frac{\zeta}{\zeta-z}+\Sigma_{1}+\overline{\Sigma_{1}},|\zeta|=r
\end{array}\right.
$$

and hence

$$
\begin{aligned}
& \frac{d \zeta}{\zeta-z}+h_{1 \bar{\zeta}}(z, \zeta) d \bar{\zeta}=\left\{\begin{array}{c}
{\left[\frac{\zeta+z}{\zeta-z}-\frac{\log |z|^{2}}{\log r^{2}}+\Sigma_{1}+\overline{\Sigma_{1}}\right] \frac{d \zeta}{\zeta}, \quad|\zeta|=1,} \\
{\left[\frac{\zeta+z}{\zeta-z}+1-\frac{\log |z|^{2}}{\log r^{2}}+\Sigma_{1}+\overline{\Sigma_{1}}\right] \frac{d \zeta}{\zeta},|\zeta|=r,}
\end{array}\right. \\
& \frac{d \zeta}{\zeta-z}-h_{1 \bar{\zeta}}(z, \zeta) d \bar{\zeta}=\left\{\begin{array}{cl}
{\left[1+\frac{\log |z|^{2}}{\log r^{2}}-\Sigma_{1}-\overline{\Sigma_{1}}\right] \frac{d \zeta}{\zeta},} & |\zeta|=1 \\
{\left[\frac{\log |z|^{2}}{\log r^{2}}-\Sigma_{1}-\overline{\Sigma_{1}}\right] \frac{d \zeta}{\zeta},} & |\zeta|=r
\end{array}\right.
\end{aligned}
$$


Substituting these relations into (3), rewriting

$$
\begin{aligned}
& \frac{1}{2 \pi i} \int_{\partial R} \operatorname{Re} w(\zeta)\left(\frac{d \zeta}{\zeta-z}+h_{1 \bar{\zeta}}(z, \zeta) d \bar{\zeta}\right)=\frac{1}{2 \pi i} \int_{\partial R} \operatorname{Re} w(\zeta)\left(\frac{\zeta+z}{\zeta-z}+2 \Sigma_{1}\right) \frac{d \zeta}{\zeta} \\
& -\frac{1}{2 \pi i} \int_{\partial R} \operatorname{Re} w(\zeta)\left(\frac{\log |z|^{2}}{\log r^{2}}+\Sigma_{1}-\overline{\Sigma_{1}}\right) \frac{d \zeta}{\zeta}-\frac{1}{2 \pi i} \int_{|\zeta|=r} \operatorname{Re} w(\zeta) \frac{d \zeta}{\zeta} \\
& \frac{1}{2 \pi} \int_{\partial R} \operatorname{Im} w(\zeta)\left(\frac{d \zeta}{\zeta-z}-h_{1 \bar{\zeta}}(z, \zeta) d \bar{\zeta}\right)=\frac{1}{2 \pi} \int_{\partial R} \operatorname{Im} w(\zeta)\left(\frac{\log |z|^{2}}{\log r^{2}}+1-\Sigma_{1}-\overline{\Sigma_{1}}\right) \frac{d \zeta}{\zeta} \\
& \quad+\frac{1}{2 \pi} \int_{|\zeta|=r} \operatorname{Im} w(\zeta) \frac{d \zeta}{\zeta}
\end{aligned}
$$

and observing

$$
\begin{aligned}
& \frac{1}{2 \pi i} \int_{\partial R} \overline{w(\zeta)} \frac{d \zeta}{\zeta}=\frac{1}{\pi} \int_{R} \frac{\overline{w_{\bar{\zeta}}(\zeta)}}{\bar{\zeta}} d \xi d \eta, \quad \frac{1}{2 \pi i} \int_{\partial R} w(\zeta) \frac{d \zeta}{\zeta}=\frac{1}{\pi} \int_{R} \frac{w_{\bar{\zeta}}(\zeta)}{\zeta} d \xi d \eta, \\
& \frac{1}{2 \pi i} \int_{\partial R} w(\zeta) \Sigma_{1} \frac{d \zeta}{\zeta}=\frac{1}{\pi} \int_{R} \frac{w_{\bar{\zeta}}(\zeta)}{\zeta} \Sigma_{1} d \xi d \eta
\end{aligned}
$$

leads to the Schwarz representation for $R$, see [28],

$$
\begin{aligned}
w(z)= & \frac{1}{2 \pi i} \int_{\partial R} \operatorname{Re} w(z)\left[\frac{\zeta+z}{\zeta-z}+2 \Sigma_{1}\right] \frac{d \zeta}{\zeta}-\frac{1}{2 \pi i} \int_{|\zeta|=r} \overline{w(\zeta)} \frac{d \zeta}{\zeta} \\
& -\frac{1}{2 \pi} \int_{R}\left\{\frac{w_{\bar{\zeta}}(\zeta)}{\zeta}\left[\frac{\zeta+z}{\zeta-z}+2 \Sigma_{1}\right]+\frac{\overline{w_{\bar{\zeta}}(\zeta)}}{\bar{\zeta}}\left[\frac{1+z \bar{\zeta}}{1-z \bar{\zeta}}+2 \Sigma_{2}\right]\right\} d \xi d \eta
\end{aligned}
$$

For general multiply connected domains the complex harmonic Green function $M_{1}(z, \zeta)$, see e.g. [25], is available for constructing the Schwarz integral operator. It may be helpful to modify the representation (3) in the general case.

\section{Polyanalytic Dirichlet problem}

While Dirichlet data for the Poisson equation provide a well-posed boundary value problem [12-14,16,21], for the Cauchy-Riemann operator they deliver an overdetermined problem. Hence, a condition is required for solvability. This situation is repeated to the Bitsadze operator, i.e. the square of the Cauchy-Riemann operator, if Dirichlet data are posed for the function and also for its $\bar{z}$-derivative, see $[6,11]$. The extension to the polyanalytic operator of arbitrary order is natural and quite simple. Just the solvability conditions have to be handled. Other boundary value problems like the Neumann $[4,27,32]$, the Robin $[14,26]$ and combinations of them for the polyanalytic (and polyharmonic) operators are, in principle, possible to be studied but turn out to be involved. Some examples were treated just for the Bitsadze operator in $[6,11]$.

In [9] the Dirichlet problem is investigated for general poly-harmonic operators in a ring domain, see also [29]. 
Definition (n-Dirichlet problem.) The iterated Dirichlet boundary value problem for the polyanalytic operator of degree $n$ is to find a solution $w$ and proper solvability conditions for the data $f, \gamma_{\mu}$ given, satisfying

$$
\partial_{\bar{z}}^{n} w(z)=f(z), \quad z \in D, \quad \partial_{\bar{\zeta}}^{\mu} w(\zeta)=\gamma_{\mu}(\zeta), \quad \zeta \in \partial D, \quad 0 \leq \mu \leq n-1,
$$

for a domain $D$ of the complex plane $\mathbb{C}$ with boundary $\partial D$.

In case a solution does exist the polyanalytic Cauchy-Pompeiu representation formula (2) provides an integral representation formula in terms of the given data [6,11]. However, the solvability conditions have to be determined.

Theorem 3 The Dirichlet problem for the polyanalytic operator with data $f \in$ $L_{p}(D ; \mathbb{C}), \quad 2<p, \quad \gamma_{\mu} \in C(\partial D ; \mathbb{C}), \quad 0 \leq \mu \leq n-1$, is solvable if and only if for $0 \leq \mu \leq n-1$,

$$
\begin{aligned}
& \frac{1}{2 \pi i} \int_{\partial D} \gamma_{\mu}(\zeta) \partial_{\zeta} h_{1}(z, \zeta) d \zeta \\
& +\sum_{\nu=\mu+1}^{n-1} \frac{1}{2 \pi i} \int_{\partial D} \gamma_{\nu}\left(\zeta_{\nu-\mu+1}\right)\left(\frac{1}{\pi} \int_{D}\right)^{\nu-\mu} \partial_{\zeta_{1}} h_{1}\left(z, \zeta_{1}\right) \prod_{\lambda=1}^{\nu-\mu} \frac{d \zeta_{\lambda} d \eta_{\lambda}}{\zeta_{\lambda}-\zeta_{\lambda+1}} d \zeta_{\nu-\mu+1} \\
& =\frac{1}{\pi} \int_{D} f\left(\zeta_{n-\mu}\right)\left(\frac{1}{\pi} \int_{D}\right)^{n-\mu-1} \partial_{\zeta_{1}} h_{1}\left(z, \zeta_{1}\right) \prod_{\lambda=1}^{n-\mu-1} \frac{d \xi_{\lambda} d \eta_{\lambda}}{\zeta_{\lambda}-\zeta_{\lambda+1}} d \xi_{n-\mu} d \eta_{n-\mu} .
\end{aligned}
$$

The solution then is given as

$$
w(z)=\sum_{\mu=0}^{n-1} \frac{1}{2 \pi i} \int_{\partial D} \frac{(-1)^{\mu}(\overline{\zeta-z})^{\mu}}{\mu !(\zeta-z)} \gamma_{\mu}(\zeta) d \zeta-\frac{1}{\pi} \int_{D} \frac{(-1)^{n-1}(\overline{\zeta-z})^{n-1}}{(n-1) !(\zeta-z)} f(\zeta) d \xi d \eta
$$

The proof is again an induction argument.

Proof For $n=1$ this is just the Dirichlet problem for the Cauchy-Riemann operator. That

$$
w(z)=\frac{1}{2 \pi i} \int_{\partial D} \gamma(\zeta) \frac{d \zeta}{\zeta-z}-\frac{1}{\pi} \int_{D} f(\zeta) \frac{d \xi d \eta}{\zeta-z}
$$

is the solution if and only if

$$
\frac{1}{2 \pi i} \int_{\partial D} \gamma(\zeta) \partial_{\zeta} h_{1}(z, \zeta) d \zeta=\frac{1}{\pi} \int_{D} f(\zeta) \partial_{\zeta} h_{1}(z, \zeta) d \xi d \eta
$$

is satisfied, is a consequence of the property of the Pompeiu operator, the Green function and the Poisson kernel. Adding the condition to the representation formula 
leads to

$$
w(z)=-\frac{1}{4 \pi} \int_{\partial D} \gamma(\zeta) \partial_{\nu_{\zeta}} G_{1}(z, \zeta) d s_{\zeta}+\frac{1}{\pi} \int_{D} f(\zeta) \partial_{\zeta} G_{1}(z, \zeta) d \xi d \eta
$$

The second part, the necessity, is a consequence of the fact that a solution to the problem $w$, in case it exists, is representable by the Cauchy-Pompeiu formula, while the function

$$
\widetilde{w}(z)=-\frac{1}{2 \pi i} \int_{\partial D} \gamma(\zeta) \partial_{\zeta} h_{1}(z, \zeta) d \zeta+\frac{1}{\pi} \int_{D} f(\zeta) \partial_{\zeta} h_{1}(z, \zeta) d \xi d \eta
$$

added to the solution $w$ obviously contributes a solution to the Dirichlet problem, $w+$ $\widetilde{w}$, for the Cauchy-Riemann equation. Hence, the harmonic function $\widetilde{w}$ has vanishing boundary values and thus is identically zero.

The uniqueness of the solution is evident from the theory of analytic functions.

To prove the statement of the theorem for $n+1$, this problem is decomposed into the system

$$
\begin{aligned}
& \partial_{\bar{z}}^{n} w=\omega \text { in } D, \quad \partial_{\bar{z}}^{\mu} w=\gamma_{\mu} \text { on } \partial D, \quad 0 \leq \mu \leq n-1 \\
& \partial_{\bar{z}} \omega=f, \quad \text { in } D, \quad \omega=\gamma_{n} \text { on } \partial D .
\end{aligned}
$$

Representing $\omega$ as

$$
\omega(z)=\frac{1}{2 \pi i} \int_{\partial D} \gamma_{n}\left(\zeta_{n-\mu+1}\right) \frac{d \zeta_{n-\mu+1}}{\zeta_{n-\mu+1}-z}-\frac{1}{\pi} \int_{D} f\left(\zeta_{n-\mu+1}\right) \frac{d \xi_{n-\mu+1} d \eta_{n-\mu+1}}{\zeta_{n-\mu+1}-z}
$$

and noticing the solvability condition

$$
\frac{1}{2 \pi i} \int_{\partial D} \gamma_{n}(\zeta) \partial_{\zeta} h_{1}(z, \zeta) d \zeta=\frac{1}{\pi} \int_{D} f(\zeta) \partial_{\zeta} h_{1}(z, \zeta) d \xi d \eta
$$

the right-hand side of the solvability conditions (9) with $f$ replaced by $\omega$ can be written as

$$
\begin{aligned}
& \frac{1}{\pi} \int_{D}\left[\frac{1}{2 \pi i} \int_{D} \gamma_{n}\left(\zeta_{n-\mu+1}\right) \frac{d \zeta_{n-\mu+1}}{\zeta_{n-\mu+1}-\zeta_{n-\mu}}\right] \\
& \times\left(\frac{1}{\pi} \int_{D}\right)^{n-\mu-1} \partial_{\zeta_{1}} h_{1}\left(z, \zeta_{1}\right) \prod_{\lambda=1}^{n-\mu-1} \frac{d \xi_{\lambda} d \eta_{\lambda}}{\zeta_{\lambda}-\zeta_{\lambda+1}} d \xi_{n-\mu} d \eta_{n-\mu} \\
& -\frac{1}{\pi} \int_{D}\left[\frac{1}{\pi} f\left(\zeta_{n-\mu+1}\right) \frac{d \xi_{n-\mu+1} d \eta_{n-\mu+1}}{\zeta_{n-\mu+1}-\zeta_{n-\mu}}\right] \\
& \times\left(\frac{1}{\pi} \int_{D}\right)^{n-\mu-1} \partial_{\zeta_{1}} h_{1}\left(z, \zeta_{1}\right) \prod_{\lambda=1}^{n-\mu-1} \frac{d \xi_{\lambda} d \eta_{\lambda}}{\zeta_{\lambda}-\zeta_{\lambda+1}} d \xi_{n-\mu} d \eta_{n-\mu}
\end{aligned}
$$


But this is just

$$
\begin{aligned}
-\frac{1}{2 \pi i}\left\{\int_{\partial D} \gamma_{n}\left(\zeta_{n-\mu+1}\right)\left(\frac{1}{\pi} \int_{D}\right)^{n-\mu} \partial_{\zeta_{1}} h_{1}\left(z, \zeta_{1}\right)\right. \\
\left.\quad \times \prod_{\lambda=1}^{n-\mu-1} \frac{d \xi_{\lambda} d \eta_{\lambda}}{\zeta_{\lambda}-\zeta_{\lambda+1}} \frac{d \xi_{n-\mu} d \eta_{n-\mu}}{\zeta_{n-\mu}-\zeta_{n-\mu+1}}\right\} d \zeta_{n-\mu+1} \\
+\frac{1}{\pi} \int_{D}\left\{f\left(\zeta_{n-\mu+1}\right)\left(\frac{1}{\pi} \int_{D}\right)^{n-\mu} \partial_{\zeta_{1}} h_{1}\left(z, \zeta_{1}\right)\right. \\
\left.\quad \times \prod_{\lambda=1}^{n-\mu-1} \frac{d \xi_{\lambda} d \eta_{\lambda}}{\zeta_{\lambda}-\zeta_{\lambda+1}} \frac{d \xi_{n-\mu} d \eta_{n-\mu}}{\zeta_{n-\mu}-\zeta_{n-\mu+1}}\right\} d \xi_{n-\mu+1} d \eta_{n-\mu+1}
\end{aligned}
$$

Replacing the area integral on the right-hand side of (9), with $f$ replaced by $\omega$, by this sum, the resulting equations supplemented with (12) form the solvability condition (9) for $n+1$.

\section{Tri-analytic Neumann problem}

Basic is the Neumann problem for the Cauchy-Riemann equation.

Theorem 4 The Neumann boundary value problem

$$
\partial_{\nu} w=\gamma \text { on } \partial D, w\left(z_{0}\right)=c, z_{0} \in D, \gamma \in C(\partial D ; \mathbb{C}), c \in \mathbb{C},
$$

for the Cauchy-Riemann equation

$$
w_{\bar{z}}=f \text { in } D, f \in C^{\alpha}(\bar{D} ; \mathbb{C}), 0<\alpha<1 \text {, }
$$

is solvable if and only if

$$
\frac{1}{2 \pi} \int_{\partial D} \gamma(\zeta) h_{1 \zeta}(z, \zeta) d s_{\zeta}+\frac{1}{2 \pi i} \int_{\partial D} f(\zeta) h_{1 \zeta}(z, \zeta) d \bar{\zeta}+\frac{1}{\pi} \int_{D} f(\zeta) h_{1 \zeta \zeta}(z, \zeta) d \xi d \eta=0 .
$$

The solution then is

$$
\begin{aligned}
w(z)= & c-\frac{1}{2 \pi} \int_{\partial D} \gamma(\zeta) \log \frac{\zeta-z}{\zeta-z_{0}} d s_{\zeta}-\frac{1}{2 \pi i} \int_{\partial D} f(\zeta) \log \frac{\zeta-z}{\zeta-z_{0}} d \bar{\zeta} \\
& -\frac{1}{\pi} \int_{D} f(\zeta) \frac{z-z_{0}}{(\zeta-z)\left(\zeta-z_{0}\right)} d \xi d \eta
\end{aligned}
$$

Proof For the proof instead of reducing the problem to the Dirichlet problem for analytic functions the result can be just verified. Obviously, the function (13) satisfies 
as well the side condition $w\left(z_{0}\right)=c$ as the differential equation $w_{\bar{z}}=f$ because of the property of the Pompeiu operator

$$
T f(z)=-\frac{1}{\pi} \int_{D} f(\zeta) \frac{d \xi d \eta}{\zeta-z}
$$

see e.g. [10,31]. For verifying the boundary condition the solvability condition is needed. The outward normal derivative $\partial_{\nu_{\zeta}}$ on the boundary $\partial D$ is expressed as $i \partial_{\nu_{\zeta}} d s=\partial_{\zeta} d \zeta-\partial_{\bar{\zeta}} d \bar{\zeta}$. Applied to the Green function $G_{1}(z, \zeta)=-\log |\zeta-z|^{2}+$ $h_{1}(z, \zeta)$, observing $(5)$, gives

$$
2\left[\frac{1}{\zeta-z}-h_{1 \zeta}(z, \zeta)\right] d \zeta=-i \partial_{\nu_{\zeta}} G_{1}(z, \zeta) d s_{\zeta}=2 i p_{1}(z, \zeta) d s_{\zeta}
$$

with the Poisson kernel $p_{1}(z, \zeta)$ of the domain $D$, satisfying

$$
\lim _{z \rightarrow \zeta_{0} \in \partial D} \frac{1}{2 \pi} \int_{\partial D} \gamma(\zeta) p_{1}(z, \zeta) d s_{\zeta}=\gamma\left(\zeta_{0}\right)
$$

for continuous $\gamma$.

Denoting $z^{\cdot}=\partial_{s} z$ a calculation from (13) shows

$$
\begin{aligned}
& w_{z}(z) z^{\cdot}-w_{\bar{z}}(z) \overline{z^{*}} \\
& =\frac{1}{2 \pi} \int_{\partial D} \gamma(\zeta) \frac{d s_{\zeta}}{\zeta-z} z^{\cdot}+\frac{1}{2 \pi i} \int_{\partial D} f(\zeta) \frac{d \bar{\zeta}}{\zeta-z} z+\Pi f(z) z-f(z) \bar{z}
\end{aligned}
$$

with the Ahlfors-Beurling operator [10,31]

$$
\Pi f(z)=-\frac{1}{\pi} \int_{D} \frac{f(\zeta)}{(\zeta-z)^{2}} d \xi d \eta
$$

Thus the solvability condition gives

$$
\begin{aligned}
& w_{z}(z) z^{\cdot}-w_{\bar{z}}(z) \overline{z^{*}} \\
& =\frac{1}{2 \pi} \int_{\partial D} \frac{i \gamma(\zeta)}{\zeta^{\cdot}(s)} p_{1}(z, \zeta) d s_{\zeta} z^{\cdot}+\frac{1}{2 \pi i} \int_{\partial D} i f(\zeta) p_{1}(z, \zeta) \frac{\overline{\zeta^{*}(s)}}{\zeta^{\cdot}(s)} d s_{\zeta} z^{\prime} \\
& -f(z) \overline{z^{*}}+\frac{1}{\pi} \int_{D} f(\zeta) \partial_{\zeta}^{2} G_{1}(z, \zeta) d \xi d \eta z^{.}
\end{aligned}
$$

Letting $z$ approach a boundary point $\zeta_{0}$ leads to

$$
i \partial_{\nu_{\zeta}} w\left(\zeta_{0}\right)=\lim _{z \rightarrow \zeta_{0}}\left[w_{z}(z) z-w_{\bar{z}}(z) \overline{z^{*}}\right]=i \gamma\left(\zeta_{0}\right)
$$


Remark 3 It is just because of the properties of the $\Pi$-operator, see [31], and the appearance of the function $f$ in some boundary integral that $f$ is required to be Hölder-continuous on the closure $\bar{D}$ of the domain $D$.

On the other hand, assuming the Neumann problem has a solution, proper manipulations of the Cauchy-Pompeiu representation formula, the Dirichlet problem for analytic functions, and an integration show that the solution has the form (13). Then necessarily the solvability condition holds.

An iteration process leads to the solution of a Neumann problem for the Bitsadze equation $[6,7]$.

Theorem 5 The iterated Neumann problem for the Bitsadze equation in a domain $D$ with Green function $G_{1}(z, \zeta)$

$w_{\overline{z z}}=f$ in $D, \quad \partial_{\nu} w=\gamma_{0}, \partial_{\nu} w_{\bar{z}}=\gamma_{1}$ on $\partial D, \quad w\left(z_{0}\right)=c_{0}, w_{\bar{z}}\left(z_{0}\right)=c_{1}, \quad z_{0} \in D$,

for

$$
f \in C^{\alpha}(\bar{D} ; \mathbb{C}), 0<\alpha<1, \quad \gamma_{0}, \gamma_{1} \in C(\partial D ; \mathbb{C}), \quad c_{0}, c_{1} \in \mathbb{C}
$$

is solvable if and only if

$$
\begin{aligned}
& \frac{1}{2 \pi} \int_{\partial D} \gamma_{0}(\zeta) h_{1 \zeta}(z, \zeta) d s_{\zeta}+c_{1}\left[\frac{1}{2 \pi i} \int_{\partial D} h_{1 \zeta}(z, \zeta) d \bar{\zeta}+\frac{1}{\pi} \int_{D} h_{1 \zeta \zeta}(z, \zeta) d \xi d \eta\right] \\
& -\frac{1}{2 \pi} \int_{\partial D} \gamma_{1}(\zeta)\left[\frac{1}{2 \pi i} \int_{\partial D} \log \frac{\zeta-\widetilde{\zeta}}{\zeta-z_{0}} h_{1 \widetilde{\zeta}}(z, \widetilde{\zeta}) d \widetilde{\zeta}+\frac{1}{\pi} \int_{D} \log \frac{\zeta-\widetilde{\zeta}}{\zeta-z_{0}} h_{1 \widetilde{\zeta} \widetilde{\zeta}}(z, \widetilde{\zeta}) d \tilde{\xi} d \tilde{\eta}\right] d s_{\zeta} \\
& -\frac{1}{2 \pi i} \int_{\partial D} f(\zeta)\left[\frac{1}{2 \pi i} \int_{\partial D} \log \frac{\zeta-\widetilde{\zeta}}{\zeta-z_{0}} h_{1 \widetilde{\zeta}}(z, \widetilde{\zeta}) d \bar{\zeta}+\frac{1}{\pi} \int_{D} \log \frac{\zeta-\widetilde{\zeta}}{\zeta-z_{0}} h_{1 \widetilde{\zeta} \widetilde{\zeta}}(z, \widetilde{\zeta}) d \tilde{\xi} d \tilde{\eta}\right] d \bar{\zeta} \\
& -\frac{1}{\pi} \int_{D} f(\zeta)\left[\frac{1}{2 \pi i} \int_{\partial D}\left(\frac{1}{\zeta-\widetilde{\zeta}}-\frac{1}{\zeta-z_{0}}\right) h_{1 \widetilde{\zeta}}(z, \widetilde{\zeta}) d \widetilde{\zeta}\right. \\
& \left.+\frac{1}{\pi} \int_{D}\left(\frac{1}{\zeta-\widetilde{\zeta}}-\frac{1}{\zeta-z_{0}}\right) h_{1 \widetilde{\zeta} \widetilde{\zeta}}(z, \widetilde{\zeta}) d \widetilde{\xi} d \tilde{\eta}\right] d \xi d \eta=0, \\
& \frac{1}{2 \pi} \int_{\partial D} \gamma_{1}(\zeta) h_{1 \zeta}(z, \zeta) d s_{\zeta}+\frac{1}{2 \pi i} \int_{\partial D} f(\zeta) h_{1 \zeta}(z, \zeta) d \bar{\zeta}+\frac{1}{\pi} \int_{D} f(\zeta) h_{1 \zeta \zeta}(z, \zeta) d \xi d \eta=0 .
\end{aligned}
$$

The solution then is

$w(z)$

$$
\begin{aligned}
& =c_{0}-\frac{c_{1}}{2 \pi i} \int_{\partial D}\left[\left(\frac{\overline{\zeta-z}}{\zeta-z}-\frac{\overline{\zeta-z_{0}}}{\zeta-z_{0}}\right) d \zeta+\log \frac{\zeta-z}{\zeta-z_{0}} d \bar{\zeta}\right]-\frac{1}{2 \pi} \int_{\partial D} \gamma_{0}(\zeta) \log \frac{\zeta-z}{\zeta-z_{0}} d s_{\zeta} \\
& +\frac{1}{2 \pi} \int_{\partial D} \gamma_{1}(\zeta) \frac{1}{2 \pi i} \int_{\partial D}\left[\left(\frac{\widetilde{\zeta}-z}{\widetilde{\zeta}-z}-\frac{\widetilde{\zeta}-z_{0}}{\widetilde{\zeta}-z_{0}}\right) \log \frac{\zeta-\widetilde{\zeta}}{\zeta-z_{0}} d \tilde{\zeta}+\log \frac{\widetilde{\zeta}-z}{\widetilde{\zeta}-z_{0}} \log \frac{\zeta-\widetilde{\zeta}}{\zeta-z_{0}} d \widetilde{\zeta}\right] d s_{\zeta} \\
& +\frac{1}{2 \pi i} \int_{\partial D} f(\zeta) \frac{1}{2 \pi i} \int_{\partial D}\left[\left(\frac{\widetilde{\zeta}-z}{\widetilde{\zeta}-z}-\frac{\widetilde{\zeta}-z_{0}}{\widetilde{\zeta}-z_{0}}\right) \log \frac{\zeta-\widetilde{\zeta}}{\zeta-z_{0}} d \widetilde{\zeta}+\log \frac{\widetilde{\zeta}-z}{\widetilde{\zeta}-z_{0}} \log \frac{\zeta-\widetilde{\zeta}}{\zeta-z_{0}} d \widetilde{\zeta}\right] d \bar{\zeta}
\end{aligned}
$$




$$
\begin{aligned}
& -\frac{1}{\pi} \int_{D} f(\zeta) \frac{1}{2 \pi i} \int_{\partial D}\left[\frac{z-z_{0}}{(\zeta-z)\left(\zeta-z_{0}\right)}\left(\frac{\widetilde{\zeta}-\zeta}{\widetilde{\zeta}-\zeta}-\frac{\widetilde{\zeta}-z}{\widetilde{\zeta}-z}\right) d \widetilde{\zeta}\right. \\
& \left.-\frac{\widetilde{\zeta}-z_{0}}{(\zeta-\widetilde{\zeta})\left(\zeta-z_{0}\right)} \log \frac{\widetilde{\zeta}-z}{\widetilde{\zeta}-z_{0}} d \overline{\widetilde{\zeta}}\right] d \xi d \eta .
\end{aligned}
$$

This is a modification of the respective Theorem 8 from [6]. It shows a nice symmetry in its four parts. The proof is based on the decomposition of the problem into a system of two Neumann problems for Cauchy-Riemann equations and Theorem 4. As for Theorem 4 also here a verification is performed.

Proof Obviously, $w\left(z_{0}\right)=c_{0}$. By differentiations

$$
\begin{aligned}
w_{\bar{z}}(z)= & c_{1}-\frac{1}{2 \pi} \int_{\partial D} \gamma_{1}(\zeta) \log \frac{\zeta-z}{\zeta-z_{0}} d s_{\zeta}-\frac{1}{2 \pi i} \int_{\partial D} f(\zeta) \log \frac{\zeta-z}{\zeta-z_{0}} d \bar{\zeta} \\
& -\frac{1}{\pi} \int_{D} f(\zeta) \frac{z-z_{0}}{(\zeta-z)\left(\zeta-z_{0}\right)} d \xi d \eta
\end{aligned}
$$

and $w_{\overline{z z}}(z)=f(z)$ are seen. From Theorem 4 it is clear that the Neumann and side conditions are satisfied for $w_{\bar{z}}$. For verifying the Neumann condition for $w$, the difference

$$
\begin{aligned}
& w_{z}(z) z^{\cdot}-w_{\bar{z}}(z) \overline{z^{*}} \\
& =-c_{1} z^{\cdot}\left[\frac{1}{\pi} \int_{D} \frac{d \xi d \eta}{(\zeta-z)^{2}}-\frac{1}{2 \pi i} \int_{\partial D} \frac{d \bar{\zeta}}{\zeta-z}\right]+\frac{z}{2 \pi} \int_{\partial D} \gamma_{0}(\zeta) \frac{d s_{\zeta}}{\zeta-z} \\
& +\frac{z}{2 \pi} \int_{\partial D} \gamma_{1}(\zeta)\left[\frac{1}{\pi} \int_{D} \log \frac{\zeta-\widetilde{\zeta}}{\zeta-z_{0}} \frac{d \widetilde{\xi} d \widetilde{\eta}}{(\widetilde{\zeta}-z)^{2}}-\frac{1}{2 \pi i} \int_{\partial D} \log \frac{\zeta-\widetilde{\zeta}}{\zeta-z_{0}} \frac{d \widetilde{\zeta}}{\widetilde{\zeta}-z}\right] d s_{\zeta} \\
& +\frac{z}{2 \pi i} \int_{\partial D} f(\zeta)\left[\frac{1}{\pi} \int_{D} \log \frac{\zeta-\widetilde{\zeta}}{\zeta-z_{0}} \frac{d \widetilde{\zeta} d \tilde{\eta}}{(\widetilde{\zeta}-z)^{2}}-\frac{1}{2 \pi i} \int_{\partial D} \log \frac{\zeta-\widetilde{\zeta}}{\zeta-z_{0}} \frac{d \widetilde{\zeta}}{\widetilde{\zeta}}\right] d \bar{\zeta} \\
& +\frac{z}{\pi} \int_{D} f(\zeta)\left[\frac{1}{\pi} \int_{D} \frac{\widetilde{\zeta}-z_{0}}{\left(\zeta-\widetilde{\zeta}\left(\zeta-z_{0}\right)\right.} \frac{d \widetilde{\xi} d \widetilde{\eta}}{(\widetilde{\zeta}-z)^{2}}-\frac{1}{2 \pi i} \int_{\partial D} \frac{\widetilde{\zeta}-z_{0}}{(\zeta-\widetilde{\zeta})\left(\zeta-z_{0}\right)} \frac{d \widetilde{\zeta}-z}{\widetilde{z}}\right] d \xi d \eta \\
& -\bar{z} c_{1}+\frac{\bar{z}}{2 \pi} \int_{\partial D} \gamma_{1}(\zeta) \log \frac{\zeta-z}{\zeta-z_{0}} d s_{\zeta}+\frac{\zeta(\zeta) \log \frac{\zeta-z}{2 \pi i} d \bar{\zeta}}{\partial-z_{0}} \int_{\partial D} \\
& +\frac{\bar{z}}{\pi} \int_{D} f(\zeta) \frac{z-z_{0}}{(\zeta-z)\left(\zeta-z_{0}\right)} d \xi d \eta
\end{aligned}
$$

is calculated. Adding the first solvability condition to this expression gives

$$
\begin{aligned}
& w_{z}(z) z^{\cdot}-w_{\bar{z}}(z) \overline{z^{*}} \\
& =-c_{1} \cdot\left[\frac{1}{\pi} \int_{D}\left(\frac{1}{(\zeta-z)^{2}}+h_{1 \zeta \zeta}(z, \zeta)\right) d \xi d \eta-\frac{1}{2 \pi i} \int_{\partial D}\left(\frac{1}{\zeta-z}-h_{1 \zeta}(z, \zeta)\right) d \bar{\zeta}\right] \\
& +\frac{z}{2 \pi} \int_{\partial D} \gamma_{0}(\zeta)\left(\frac{1}{\zeta-z}-h_{1 \zeta}(z, \zeta)\right) d s_{\zeta} \\
& +\frac{z}{2 \pi} \int_{\partial D} f(\zeta)\left[\frac{1}{\pi} \int_{D} \log \frac{\zeta-\widetilde{\zeta}}{\zeta-z_{0}}\left(\frac{1}{(\widetilde{\zeta}-z)^{2}}+h_{1 \widetilde{\zeta} \widetilde{\zeta}}(z, \widetilde{\zeta})\right) d \widetilde{\xi} d \tilde{\eta}\right.
\end{aligned}
$$




$$
\begin{aligned}
& \left.-\frac{1}{2 \pi i} \int_{\partial D} \log \frac{\zeta-\widetilde{\zeta}}{\zeta-z_{0}}\left(\frac{1}{\widetilde{\zeta}-z}-h_{1 \widetilde{\zeta}}(z, \widetilde{\zeta})\right) d \widetilde{\zeta}\right] d \bar{\zeta} \\
& +\frac{z}{\pi} \int_{D} f(\zeta)\left[\frac{1}{\pi} \int_{D} \frac{\tilde{\zeta}-z_{0}}{\left(\zeta-\widetilde{\zeta}\left(\zeta-z_{0}\right)\right.}\left(\frac{1}{(\widetilde{\zeta}-z)^{2}}+h_{1 \widetilde{\zeta} \widetilde{\zeta}}(z, \widetilde{\zeta})\right) d \widetilde{\xi} d \widetilde{\eta}\right. \\
& \left.-\frac{1}{2 \pi i} \int_{\partial D} \frac{\tilde{\zeta}-z_{0}}{(\zeta-\widetilde{\zeta})\left(\zeta-z_{0}\right)}\left(\frac{1}{\widetilde{\zeta}-z}-h_{1 \widetilde{\zeta}}(z, \widetilde{\zeta})\right) d \bar{\zeta}\right] d \xi d \eta \\
& -\bar{z} c_{1}+\frac{\bar{z}}{2 \pi} \int_{\partial D} \gamma_{1}(\zeta) \log \frac{\zeta-z}{\zeta-z_{0}} d s_{\zeta}+\frac{\bar{z}}{2 \pi i} \int_{\partial D} f(\zeta) \log \frac{\zeta-z}{\zeta-z_{0}} d \bar{\zeta} \\
& +\frac{\bar{z}}{\pi} \int_{D} f(\zeta) \frac{z-z_{0}}{(\zeta-z)\left(\zeta-z_{0}\right)} d \xi d \eta .
\end{aligned}
$$

Observing the property of the Poisson kernel and the fact that $G_{1}(z, \zeta)$ vanishes identically in $\zeta$ for $z \in \partial D$,

$$
\lim _{z \rightarrow \zeta \in \partial D}\left[w_{z}(z) z^{\cdot}-w_{\bar{z}}(z) \overline{z^{*}}\right]=i \gamma_{0}(\zeta)
$$

is seen.

Corollary 1 The iterated Neumann problem for the Bitsadze equation is solvable if and only if

$$
\begin{aligned}
& \frac{1}{2 \pi} \int_{\partial D} \gamma_{0}(\zeta) h_{1 \zeta}(z, \zeta) d s_{\zeta}-\frac{1}{2 \pi} \int_{\partial D} \gamma_{1}(\zeta) \frac{1}{\pi} \int_{D} h_{1}(z, \widetilde{\zeta}) \frac{d \widetilde{\xi} d \widetilde{\eta}}{\widetilde{\zeta}-\zeta} d s_{\zeta} \\
& -\frac{1}{2 \pi i} \int_{\partial D} f(\zeta) \frac{1}{\pi} \int_{D} h_{1 \widetilde{\zeta}}(z, \widetilde{\zeta}) \frac{d \widetilde{\xi} d \widetilde{\eta}}{\widetilde{\zeta}-\zeta} d \bar{\zeta} \\
& -\frac{1}{\pi} \int_{D} f(\zeta) \frac{1}{\pi} \int_{D} h_{1 \widetilde{\zeta}}(z, \widetilde{\zeta}) \frac{d \widetilde{\xi} d \widetilde{\eta}}{(\widetilde{\zeta}-\zeta)^{2}} d \xi d \eta=0, \\
& \frac{1}{2 \pi} \int_{\partial D} \gamma_{1}(\zeta) h_{1 \zeta}(z, \zeta) d s_{\zeta}+\frac{1}{2 \pi i} \int_{\partial D} f(\zeta) h_{1 \zeta}(z, \zeta) d \bar{\zeta}+\frac{1}{\pi} \int_{D} f(\zeta) h_{1 \zeta \zeta}(z, \zeta) d \xi d \eta=0 .
\end{aligned}
$$

The solution then is

$$
\begin{aligned}
w(z)= & c_{0}+c_{1}\left(\overline{z-z_{0}}\right)-\frac{1}{2 \pi} \int_{\partial D} \gamma_{0}(\zeta) \log \frac{\zeta-z}{\zeta-z_{0}} d s_{\zeta} \\
& -\frac{1}{2 \pi} \int_{\partial D} \gamma_{1}(\zeta) \frac{1}{2 \pi i} \int_{\partial D} \frac{\widetilde{\zeta}-\zeta}{\widetilde{\zeta}-\zeta} \log \frac{\widetilde{\zeta}-z}{\widetilde{\zeta}-z_{0}} d \widetilde{\zeta} d s_{\zeta} \\
& -\frac{1}{2 \pi i} \int_{\partial D} f(\zeta) \frac{1}{2 \pi i} \int_{\partial D} \frac{\widetilde{\zeta}-\zeta}{\widetilde{\zeta}-\zeta} \log \frac{\widetilde{\zeta}-z}{\widetilde{\zeta}-z_{0}} d \widetilde{\zeta} d \bar{\zeta} \\
& -\frac{1}{\pi} \int_{D} f(\zeta) \frac{1}{2 \pi i} \int_{\partial D} \frac{\frac{\zeta}{\widetilde{\zeta}-\zeta}}{\widetilde{(\widetilde{\zeta}-\zeta)^{2}}} \log \frac{\widetilde{\zeta}-z}{\widetilde{\zeta}-z_{0}} d \widetilde{\zeta} d \xi d \eta
\end{aligned}
$$


Proof Observing the relation

$$
\frac{1}{2 \pi i} \int_{\partial D} \frac{\bar{\zeta}-z}{\widetilde{\zeta}-z} d \widetilde{\zeta}=\frac{1}{\pi} \int_{D} \frac{d \widetilde{\xi} d \widetilde{\eta}}{\widetilde{\zeta}-z}
$$

the factor of $-c_{1}$ in the formula for $w$ in Theorem 5 by integration by parts and the Cauchy-Pompeiu formula is seen to be

$$
\frac{1}{2 \pi i} \int_{\partial D} \log \frac{\widetilde{\zeta}-z}{\widetilde{\zeta}-z_{0}} d \overline{\widetilde{\zeta}}+\frac{1}{\pi} \int_{D}\left[\frac{1}{\widetilde{\zeta}-z}-\frac{1}{\widetilde{\zeta}-z_{0}}\right] d \widetilde{\xi} d \widetilde{\eta}=-\left(\overline{z-z_{0}}\right)
$$

Also the Gauss theorem and the Cauchy-Pompeiu formula imply

$$
\begin{aligned}
& \frac{1}{2 \pi i} \int_{\partial D}\left[\left(\frac{\widetilde{\zeta}-z}{\widetilde{\zeta}-z}-\frac{\widetilde{\zeta}-z_{0}}{\widetilde{\zeta}-z_{0}}\right) \log \frac{\zeta-\widetilde{\zeta}}{\zeta-z_{0}} d \widetilde{\zeta}+\log \frac{\widetilde{\zeta}-z}{\widetilde{\zeta}-z_{0}} \log \frac{\zeta-\widetilde{\zeta}}{\zeta-z_{0}} d \widetilde{\zeta}\right] \\
& =-\frac{1}{\pi} \int_{D} \log \frac{\widetilde{\zeta}-z}{\widetilde{\zeta}-z_{0}} \frac{d \widetilde{\zeta} d \tilde{\zeta}}{\widetilde{\zeta}-\zeta}=-\frac{1}{2 \pi i} \int_{\partial D} \frac{\widetilde{\zeta}-\zeta}{\widetilde{\zeta}-\zeta} \log \frac{\widetilde{\zeta}-z}{\widetilde{\zeta}-z_{0}} d \widetilde{\zeta}, \\
& \frac{1}{2 \pi i} \int_{\partial D}\left[\frac{z-z_{0}}{(\zeta-z)\left(\zeta-z_{0}\right)}\left(\frac{\widetilde{\zeta}-\zeta}{\widetilde{\zeta}-\zeta}-\frac{\widetilde{\zeta}-z}{\widetilde{\zeta}-z}\right) d \widetilde{\zeta}\right. \\
& \left.-\frac{\widetilde{\zeta}-z_{0}}{(\zeta-\widetilde{\zeta})\left(\zeta-z_{0}\right)} \log \frac{\widetilde{\zeta}-z}{\widetilde{\zeta}-z_{0}} d \widetilde{\zeta}\right]=\frac{1}{2 \pi i} \int_{\partial D} \frac{\widetilde{\widetilde{\zeta}-\zeta}}{(\widetilde{\zeta}-\zeta)^{2}} \log \frac{\widetilde{\zeta}-z}{\widetilde{\zeta}-z_{0}} d \widetilde{\zeta} \text {. }
\end{aligned}
$$

Moreover, the first solvability condition can be simplified as

$$
\begin{aligned}
& \frac{1}{2 \pi i} \int_{\partial D} h_{1 \zeta}(z, \zeta) d \bar{\zeta}+\frac{1}{\pi} \int_{D} h_{1 \zeta \zeta}(z, \zeta) d \xi d \eta=0, \\
& \frac{1}{2 \pi i} \int_{\partial D} \log \frac{\zeta-\widetilde{\zeta}}{\zeta-z_{0}} h_{1 \widetilde{\zeta}}(z, \zeta) d \widetilde{\zeta}+\frac{1}{\pi} \int_{D} \log \frac{\zeta-\widetilde{\zeta}}{\zeta-z_{0}} h_{1 \widetilde{\zeta} \widetilde{\zeta}}(z, \zeta) d \widetilde{\xi} d \widetilde{\eta} \\
& =-\frac{1}{\pi} \int_{D} h_{1 \widetilde{\zeta}}(z, \widetilde{\zeta}) \frac{d \widetilde{\xi} d \widetilde{\eta}}{\widetilde{\zeta}-\zeta}=-\frac{1}{2 \pi i} \int_{\partial D} h_{1 \widetilde{\zeta}}(z, \widetilde{\zeta}) \underset{\widetilde{\zeta}-\zeta}{\widetilde{\zeta}-\zeta} d \widetilde{\zeta}, \\
& \frac{1}{2 \pi i} \int_{\partial D} \frac{\widetilde{\zeta}-z_{0}}{(\zeta-\widetilde{\zeta})\left(\zeta-z_{0}\right)} h_{1 \tilde{\zeta}}(z, \widetilde{\zeta}) d \widetilde{\zeta}+\frac{1}{\pi} \int_{D} \frac{\widetilde{\zeta}-z_{0}}{(\zeta-\widetilde{\zeta})\left(\zeta-z_{0}\right)} h_{1 \tilde{\zeta} \tilde{\zeta}}(z, \widetilde{\zeta}) d \tilde{\xi} d \tilde{\eta} \\
& =-\frac{1}{\pi} \int_{D} h_{1 \widetilde{\zeta}}(z, \widetilde{\zeta}) \frac{d \widetilde{\xi} d \widetilde{\eta}}{(\widetilde{\zeta}-\zeta)^{2}}=-\frac{1}{2 \pi i} \int_{\partial D} h_{1 \widetilde{\zeta}}(z, \widetilde{\zeta}) \frac{\widetilde{\zeta}-\zeta}{(\widetilde{\zeta}-\zeta)^{2}} d \widetilde{\zeta} .
\end{aligned}
$$

Continuing this iteration process, the iterated Neumann problem is treated for the tri-analytic equation.

Theorem 6 The problem

$$
w_{\overline{z z z}}=f \text { in } D, \quad \partial_{\nu} w=\gamma_{0}, \quad \partial_{\nu} w_{\bar{z}}=\gamma_{1}, \quad \partial_{\nu} w_{\overline{z z}}=\gamma_{2} \text { on } \partial D
$$




$$
\begin{aligned}
& w\left(z_{0}\right)=c_{0}, \quad w_{\bar{z}}\left(z_{0}\right)=c_{1}, \quad w_{\overline{z z}}\left(z_{0}\right)=c_{2}, \\
& \text { for } f \in C^{\alpha}(\bar{D} ; \mathbb{C}), \quad \gamma_{k} \in C(\partial D ; \mathbb{C}), \quad c_{k} \in \mathbb{C}, \quad k=1,2,3,
\end{aligned}
$$

is solvable if and only if the conditions

$$
\begin{aligned}
& \frac{1}{2 \pi} \int_{\partial D} \gamma_{0}(\zeta) h_{1 \zeta}(z, \zeta) d s_{\zeta}+\frac{1}{2 \pi} \int_{\partial D} \gamma_{1}(\zeta) \frac{1}{2 \pi i} \int_{\partial D} \frac{\overline{\widetilde{\zeta}-\zeta}}{\widetilde{\zeta}-\zeta)^{2}} h_{1}(z, \tilde{\zeta}) d \widetilde{\zeta} d s_{\zeta}
\end{aligned}
$$

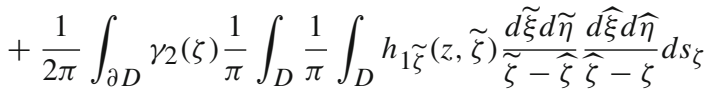

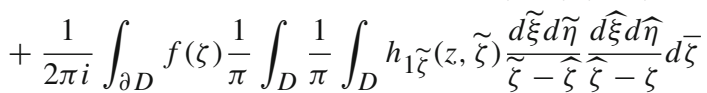

$$
\begin{aligned}
& +\frac{1}{\pi} \int_{D} f(\zeta) \frac{1}{\pi} \int_{D} \frac{1}{\pi} \int_{D} h_{1 \widetilde{\zeta}}(z, \widetilde{\zeta}) \frac{d \widetilde{\xi} d \widetilde{\eta}}{\widetilde{\zeta}-\widehat{\zeta}} \frac{d \widehat{\xi} d \widehat{\eta}}{(\widehat{\zeta}-\zeta)^{2}} d \xi d \eta=0, \\
& \frac{1}{2 \pi} \int_{\partial D} \gamma_{1}(\zeta) h_{1 \zeta}(z, \zeta) d s_{\zeta}+\frac{1}{2 \pi} \int_{\partial D} \gamma_{2}(\zeta) \frac{1}{\pi} \int_{D} h_{1 \widetilde{\zeta}}(z, \widetilde{\zeta}) \underset{\widetilde{\zeta}-\zeta}{\frac{d \tilde{\xi}}{\widetilde{\eta}}} d s_{\zeta} \\
& +\frac{1}{2 \pi i} \int_{\partial D} f(\zeta) \frac{1}{\pi} \int_{D} h_{1} \widetilde{\zeta}(z, \widetilde{\zeta}) \frac{d \widetilde{\xi} d \tilde{\eta}}{\widetilde{\zeta}-\zeta} d \bar{\zeta}+\frac{1}{\pi} \int_{D} f(\zeta) \frac{1}{\pi} \int_{D} h_{1}\left(\widetilde{\zeta}(z, \widetilde{\zeta}) \frac{d \widetilde{\xi} d \widetilde{\eta}}{(\widetilde{\zeta}-\zeta)^{2}} d \xi d \eta=0,\right. \\
& \frac{1}{2 \pi} \int_{\partial D} \gamma_{2}(\zeta) h_{1 \zeta}(z, \zeta) d s_{\zeta}+\frac{1}{2 \pi i} \int_{\partial D} f(\zeta) h_{1 \zeta}(z, \zeta) d \bar{\zeta}+\frac{1}{\pi} \int_{D} f(\zeta) h_{1 \zeta \zeta}(z, \zeta) d \xi d \eta=0
\end{aligned}
$$

are satisfied. Then the solution is

$$
\begin{aligned}
& w(z)=c_{0}+c_{1}\left(\overline{z-z_{0}}\right)+c_{2} \frac{1}{2}\left(\overline{z-z_{0}}\right)^{2}-\frac{1}{2 \pi} \int_{\partial D} \gamma_{0}(\zeta) \log \frac{\zeta-z}{\zeta-z_{0}} d s_{\zeta}
\end{aligned}
$$

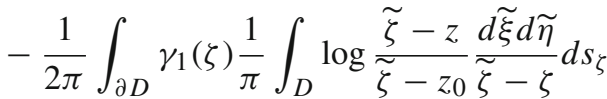

$$
\begin{aligned}
& -\frac{1}{2 \pi} \int_{\partial D} \gamma_{2}(\zeta) \frac{1}{\pi} \int_{D} \frac{1}{\pi} \int_{D} \frac{d \widetilde{\xi} d \tilde{\eta}}{\widetilde{\zeta}-\widehat{\zeta}} \log \frac{\widehat{\zeta}-z}{\widehat{\zeta}-z_{0}} \frac{d \widehat{\xi} d \widehat{\eta}-\zeta}{\widehat{\zeta}} d s_{\zeta} \\
& -\frac{1}{2 \pi i} \int_{\partial D} f(\zeta) \frac{1}{\pi} \int_{D} \frac{1}{\pi} \int_{D} \frac{d \widetilde{\xi} d \widetilde{\eta}}{\widetilde{\zeta}-\widehat{\zeta}} \log \frac{\widehat{\zeta}-z}{\widehat{\zeta}-z_{0}} \frac{d \widehat{\xi} d \widehat{\eta}}{\widehat{\zeta}-\zeta} d \bar{\zeta} \\
& -\frac{1}{\pi} \int_{D} f(\zeta) \frac{1}{\pi} \int_{D} \frac{1}{\pi} \int_{D} \log \frac{\widehat{\zeta}-z}{\widehat{\zeta}-z_{0}} \frac{d \widehat{\xi} d \widehat{\eta}}{\widehat{\zeta}} \frac{d \widetilde{\xi} d \tilde{\eta}}{(\widetilde{\zeta}-\zeta)^{2}} d \xi d \eta \text {. }
\end{aligned}
$$

Proof Decomposing the problem in a system of Neumann problems for the Bitsadze and the Cauchy-Riemann equation

$$
\begin{aligned}
& w_{\overline{z z}}=\omega \text { in } D, \partial_{\nu} w=\gamma_{0}, \partial_{\nu} w_{\bar{z}}=\gamma_{1} \text { on } \partial D, \quad w\left(z_{0}\right)=c_{0}, w_{\bar{z}}\left(z_{0}\right)=c_{1}, \\
& \omega_{\bar{z}}=f \text { in } D, \partial_{\nu} \omega=\gamma_{2} \text { on } \partial D, \quad \omega\left(z_{0}\right)=c_{2},
\end{aligned}
$$

and inserting $\omega$ in the form (13) from Theorem 4 into the solution $w$ from Theorem 5 with $\omega$ in place of $f$, give the solution in the stated form. Just Lemma 3 from the appendix is needed. The same procedure is applied to the solvability conditions. 
Remark 4 The solutions of the Neumann problem for the Bitsadze equation and for the trianalytic equation show a remarkable pattern. This gives a hint how the respective solution will look like for the n-analytic equation. But its disadvantage is that the relation to the polyanalytic equation is not unveiled. Neither is the sum of boundary integrals seen to form a polyanalytic function nor does the area integral look like a potential to the respective polyanalytic operator. In this regard, the solution in Theorem 5 is preferable to the form from Corollary 1.

In order to show the relations between the three solutions for the iterated Neumann problem for the three equations considered so far, two lemmas are exemplarily exposed. By applying integration by parts, as well the boundary integrals are recognized to be polyanalytic as the area integral is seen to be a particular solution to the respective inhomogeneous polyanalytic equation. Somehow the area integral is a higher order Pompeiu operator in relation to the Neumann conditions. The first lemma does unveil the relations of the two kernel functions in the boundary integrals in the solution from Corollary 1.

Lemma 2 For $z, \zeta, z_{0} \in D$

$$
\partial_{\bar{z}} \frac{1}{\pi} \int_{D} \log \frac{\widetilde{\zeta}-z}{\widetilde{\zeta}-z_{0}} \frac{d \widetilde{\zeta} d \tilde{\zeta}}{\widetilde{\zeta}-\zeta}=\log \frac{\zeta-z}{\zeta-z_{0}}
$$

Proof In order to apply integration by parts according to Lemma 6, the function $\log \frac{\zeta-z}{\zeta-z_{0}}$ with $\partial_{z} \log \frac{\zeta-z}{\zeta-z_{0}}=\frac{1}{z-\zeta}$ is introduced leading to

$$
\begin{aligned}
& \frac{1}{\pi} \int_{D} \log \frac{\widetilde{\zeta}-z}{\widetilde{\zeta}-z_{0}} \frac{d \widetilde{\zeta} d \widetilde{\zeta}}{\widetilde{\zeta}-\zeta}=\frac{1}{\pi} \int_{D} \log \frac{\widetilde{\zeta}-z}{\widetilde{\zeta}-z_{0}} \partial_{\widetilde{\zeta}} \log \frac{\zeta-\widetilde{\zeta}}{\zeta-z_{0}} d \widetilde{\xi} d \widetilde{\eta} \\
& =-\frac{1}{\pi} \int_{D} \log \frac{\zeta-\widetilde{\zeta}}{\zeta-z_{0}}\left[\frac{1}{\widetilde{\zeta}-z}-\frac{1}{\widetilde{\zeta}-z_{0}}\right] d \widetilde{\xi} d \tilde{\eta}-\frac{1}{2 \pi i} \int_{\partial D} \log \frac{\widetilde{\zeta}-z}{\widetilde{\zeta}-z_{0}} \log \frac{\zeta-\widetilde{\zeta}}{\zeta-z_{0}} d \widetilde{\zeta}
\end{aligned}
$$

As here the boundary integral is an analytic function in $z$, the result follows from the property of the Pompeiu operator $T$.

Remark 5 A consequence of this lemma is

$$
\partial_{\bar{z}} \frac{1}{2 \pi} \int_{\partial D} \gamma_{1}(\zeta) \frac{1}{\pi} \int_{D} \log \frac{\widetilde{\zeta}-z}{\widetilde{\zeta}-z_{0}} \frac{d \widetilde{\xi} d \tilde{\eta}}{\widetilde{\zeta}-\zeta} d s_{\zeta}=\frac{1}{2 \pi} \int_{\partial D} \gamma_{1}(\zeta) \log \frac{\zeta-z}{\zeta-z_{0}} d s_{\zeta}
$$

Rewriting the integral on the left-hand side as

$$
\frac{1}{2 \pi} \int_{\partial D} \gamma_{1}(\zeta) \frac{1}{2 \pi i} \int_{\partial D} \frac{\bar{\zeta}-\zeta}{\widetilde{\zeta}-\zeta} \log \frac{\widetilde{\zeta}-z}{\widetilde{\zeta}-z_{0}} d \widetilde{\zeta} d s_{\zeta}
$$

its $\bar{z}$-derivative is not immediately recognized to be analytic. 
The same method is used to verify that the area integral in the representation of the solution in Corollary 1 is a particular solution to the inhomogeneous Bitsadze equation.

Lemma 3 For $z, \zeta, z_{0} \in D$

$$
\partial_{\bar{z}} \frac{1}{\pi} \int_{D} \log \frac{\widetilde{\zeta}-z}{\widetilde{\zeta}-z_{0}} \frac{d \widetilde{\xi} d \widetilde{\eta}}{(\widetilde{\zeta}-\zeta)^{2}}=\frac{1}{\zeta-z}
$$

Proof The procedure is as in the previous proof. It provides

$$
\begin{aligned}
& \frac{1}{\pi} \int_{D} \log \frac{\widetilde{\zeta}-z}{\widetilde{\zeta}-z_{0}} \frac{d \widetilde{\xi} d \widetilde{\eta}}{\widetilde{\zeta}-\zeta)^{2}}=-\frac{1}{\pi} \int_{D} \log \frac{\widetilde{\zeta}-z}{\widetilde{\zeta}-z_{0}} \partial_{\widetilde{\zeta}} \frac{1}{\widetilde{\zeta}-\zeta} d \widetilde{\xi} d \widetilde{\eta} \\
& =\frac{1}{\pi} \int_{D}\left[\frac{1}{\widetilde{\zeta}-z}-\frac{1}{\widetilde{\zeta}-z_{0}}\right] \frac{d \widetilde{\xi} d \widetilde{\zeta}}{\widetilde{\zeta}-\zeta}+\frac{1}{2 \pi i} \int_{\partial D} \log \frac{\widetilde{\zeta}-z}{\widetilde{\zeta}-z_{0}} \frac{d \widetilde{\zeta}-\zeta}{\widetilde{\zeta}}
\end{aligned}
$$

While the first integral in the preceding line is just

$$
\frac{1}{\pi} \frac{1}{\zeta-z} \int_{D}\left[\frac{1}{\widetilde{\zeta}-\zeta}-\frac{1}{\widetilde{\zeta}-z}\right] d \widetilde{\xi} d \widetilde{\eta}-\frac{1}{\pi} \frac{1}{\zeta-z_{0}} \int_{D}\left[\frac{1}{\widetilde{\zeta}-\zeta}-\frac{1}{\widetilde{\zeta}-z_{0}}\right] d \widetilde{\xi} d \tilde{\eta},
$$

the second one is an analytic function of $z$. Thus again by the property of the Pompeiu operator this shows the required formula.

Remark 6 A consequence of Lemma 3 is

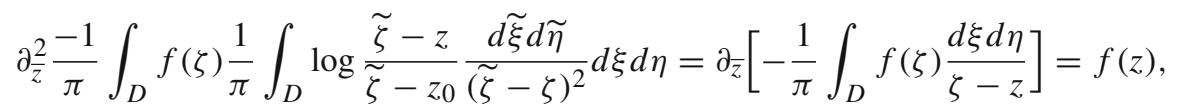

hence this area integral is a particular solution to the Bitsadze equation. It can also be written as

$$
-\frac{1}{\pi} \int_{D} f(\zeta) \frac{1}{2 \pi i} \int_{\partial D} \log \frac{\widetilde{\zeta}-z}{\widetilde{\zeta}-z_{0}} \frac{\widetilde{\zeta}-\zeta}{(\widetilde{\zeta}-\zeta)^{2}} d \tilde{\zeta} d \xi d \eta
$$

Remark 7 With respect to the trianalytic problem the same treatment results in

$$
\begin{aligned}
& \partial_{\bar{z}} \frac{1}{2 \pi} \int_{\partial D} \gamma_{2}(\zeta) \frac{1}{\pi} \int_{D} \frac{1}{\pi} \int_{D} \frac{d \widetilde{\xi} d \widetilde{\eta}}{\widetilde{\zeta}-\widehat{\zeta}} \log \frac{\widehat{\zeta}-z}{\widehat{\zeta}-z_{0}} \frac{d \widehat{\xi} d \widehat{\eta}}{\widehat{\zeta}-\zeta} d s_{\zeta} \\
& =\frac{1}{2 \pi} \int_{\partial D} \gamma_{2}(\zeta) \frac{1}{\pi} \int_{D} \log \frac{\widetilde{\zeta}-z}{\widetilde{\zeta}-z_{0}} \frac{d \widetilde{\xi} d \widetilde{\eta}}{\widetilde{\zeta}-\zeta} d s_{\zeta} .
\end{aligned}
$$

Rewriting the area integral of the solution in Theorem 6 as

$$
-\frac{1}{\pi} \int_{D} f(\zeta) \frac{1}{\pi} \int_{D} \frac{1}{\pi} \int_{D} \log \frac{\widehat{\zeta}-z}{\widehat{\zeta}-z_{0}} \frac{\widehat{d} \hat{\xi} d \widehat{\eta}}{\widehat{\zeta}} d \widetilde{\zeta}\left[\frac{1}{\zeta-\widetilde{\zeta}}\right] d \widetilde{\xi} d \widetilde{\eta} d \xi d \eta,
$$


integration by parts shows this to be equal to

$$
\begin{aligned}
& \frac{1}{\pi} \int_{D} f(\zeta) \frac{1}{\pi} \int_{D} \frac{1}{\pi} \int_{D} \log \frac{\widehat{\zeta}-z}{\widehat{\zeta}-z_{0}} d_{\widehat{\zeta}}\left[\frac{1}{\widetilde{\zeta}-\widehat{\zeta}}\right] \frac{d \widehat{\xi} d \widehat{\zeta}}{\zeta-\widetilde{\zeta}} d \widetilde{\xi} d \tilde{\eta} d \xi d \eta \\
& +\frac{1}{\pi} \int_{D} f(\zeta) \frac{1}{\pi} \int_{D} \log \frac{\widehat{\zeta}-z}{\widehat{\zeta}-z_{0}} \frac{1}{2 \pi i} \int_{\partial D} \frac{d \widetilde{\zeta}}{(\widetilde{\zeta}-\widehat{\zeta})(\zeta-\widetilde{\zeta})} d \widehat{\xi} d \widehat{\eta} d \xi d \eta
\end{aligned}
$$

Another integration by parts turns the first integral (15) into

$$
\begin{aligned}
& -\frac{1}{\pi} \int_{D} f(\zeta) \frac{1}{\pi} \int_{D} \frac{1}{\pi} \int_{D}\left[\frac{1}{\widehat{\zeta}-z}-\frac{1}{\widehat{\zeta}-z_{0}}\right] \frac{d \widehat{\xi} d \widehat{\eta}}{\widetilde{\zeta}-\widehat{\zeta})(\zeta-\widetilde{\zeta})} d \widetilde{\xi} d \tilde{\eta} d \xi d \eta \\
& -\frac{1}{\pi} \int_{D} f(\zeta) \frac{1}{2 \pi i} \int_{\partial D} \frac{1}{\pi} \int_{D} \log \frac{\widehat{\zeta}-z}{\widehat{\zeta}-z_{0}} \frac{d \widehat{\zeta}-\widehat{\zeta}}{\widetilde{\zeta}} \frac{d \widetilde{\xi} d \widetilde{\eta}}{\zeta-\widetilde{\zeta}} d \xi d \eta .
\end{aligned}
$$

Replacing in the second integral (16) $\frac{1}{\zeta-\widehat{\zeta}}$ by $-d_{\widehat{\zeta}} \log \frac{\widetilde{\zeta}-\widehat{\zeta}}{\zeta-z_{0}}$, partial integration once again gives

$$
\begin{aligned}
& \frac{1}{\pi} \int_{D} \frac{1}{2 \pi i} \int_{\partial D} f(\zeta) \frac{1}{\pi} \int_{D}\left[\frac{1}{\widehat{\zeta}-z}-\frac{1}{\widehat{\zeta}-z_{0}}\right] \log \frac{\widetilde{\zeta}-\widehat{\zeta}-z_{0}}{\widetilde{\zeta}} d \widehat{\eta} \frac{d \bar{\zeta}}{\zeta-\widetilde{\zeta}} d \xi d \eta \\
& +\frac{1}{\pi} \int_{D} f(\zeta) \frac{1}{2 \pi i} \int_{\partial D} \frac{1}{2 \pi i} \int_{\partial D} \log \frac{\widehat{\zeta}-z}{\widehat{\zeta}-z_{0}} \log \frac{\widetilde{\zeta}-\widehat{\zeta}}{\widetilde{\zeta}-z_{0}} d \bar{\zeta} \frac{d \widetilde{\zeta}}{\zeta-\widetilde{\zeta}} d \xi d \eta
\end{aligned}
$$

As the integrals (18) and (20) are analytic and the integral (19), because its $z$-bar derivative is

$$
\frac{1}{\pi} \int_{D} f(\zeta) \frac{1}{2 \pi i} \int_{\partial D} \log \frac{\widetilde{\zeta}-z}{\widetilde{\zeta}-z_{0}} \frac{d \widetilde{\zeta}}{\widetilde{\zeta}-\zeta} d \xi d \eta
$$

is bianalytic, then the integral (16) is bianalytic and (14) is up to a bianalytic function equal to (17). Applying the Bitsadze operator $\partial_{\bar{z}}^{2}$ to (15) results in the Pompeiu operator:

$$
\begin{aligned}
& \partial_{\bar{z}} \frac{-1}{\pi} \int_{D} f(\zeta) \frac{1}{\pi} \int_{D} \frac{1}{\pi} \int_{D}\left[\frac{1}{\widehat{\zeta}-z}-\frac{1}{\widehat{\zeta}-z_{0}}\right] \frac{d \widehat{\xi} d \widehat{\eta}}{(\widetilde{\zeta}-\widehat{\zeta})(\zeta-\widetilde{\zeta})} d \tilde{\xi} d \tilde{\eta} d \xi d \eta \\
& =\partial_{\bar{z}} \frac{1}{\pi} \int_{D} f(\zeta) \frac{1}{\pi} \int_{D} \frac{d \widetilde{\xi} d \widetilde{\eta}}{(\widetilde{\zeta}-z)(\zeta-\widetilde{\zeta})} d \xi d \eta=-\frac{1}{\pi} \int_{D} f(\zeta) \frac{d \xi d \eta}{\zeta-z}
\end{aligned}
$$

Hence (14) is shown to be a particular solution to the inhomogeneous trianalytic equation $\partial_{\frac{3}{z}} w=f$.

The preceding remark gives a hint for the expected form of solutions to iterated Neumann problems for polyanalytic operators. The obstacle is the form of the solution. Besides also the solvability conditions need to be observed. This topic is preserved for the future. 


\section{Appendix(Technical Lemmas)}

Lemma 4 For $f, g, h \in C^{1}(D ; \mathbb{C}) \cap C(\bar{D} ; \mathbb{C})$ with $f_{\bar{z}}=0, g_{\bar{z}}=h_{z}$ in $D$ the relation

$$
\frac{1}{2 \pi i} \int_{\partial D} f[g d z+h d \bar{z}]=-\frac{1}{\pi} \int_{D} f_{z} h d x d y
$$

holds.

Lemma 5 (integration by parts.) For $f, g \in C^{1}(D ; \mathbb{C}) \cap C(\bar{D} ; \mathbb{C})$

$$
\begin{aligned}
& \frac{1}{\pi} \int_{D} f_{z} g d x d y=-\frac{1}{\pi} \int_{D} f g_{z} d x d y-\frac{1}{2 \pi i} \int_{\partial D} f g d \bar{z}, \\
& \frac{1}{\pi} \int_{D} f_{\bar{z}} g d x d y=-\frac{1}{\pi} \int_{D} f g_{\bar{z}} d x d y+\frac{1}{2 \pi i} \int_{\partial D} f g d z
\end{aligned}
$$

are valid.

\section{Corollary 2 The equation}

$$
\frac{1}{\pi} \int_{D} \frac{d x d y}{\left(z-z_{1}\right)^{2}\left(z-z_{2}\right)}=-\frac{1}{\pi} \int_{D} \frac{d x d y}{\left(z-z_{1}\right)\left(z-z_{2}\right)^{2}}+\frac{1}{2 \pi i} \int_{\partial D} \frac{d \bar{z}}{\left(z-z_{1}\right)\left(z-z_{2}\right)}
$$

holds for any $z_{1}, z_{2} \in \mathbb{C}$.

The proofs are just applications of the Gauss theorem.

Acknowledgements Both authors are grateful to editor and referees for their critical and helpful comments.

Funding Open Access funding enabled and organized by Projekt DEAL. The second named author was supported by Ministry of Education and Science of the Republic of Kazakhstan Grant No AP08052345 and in the fall term 2019 at FU Berlin through the Re-Invitation Programme for Former Scholarship Holders, 2019 (57440919) of the German Academic Exchange Service (DAAD).

Data Availability The manuscript has no associated data.

\section{Declarations}

Conflict of Interest The authors declare that they have no conflict of interest.

Open Access This article is licensed under a Creative Commons Attribution 4.0 International License, which permits use, sharing, adaptation, distribution and reproduction in any medium or format, as long as you give appropriate credit to the original author(s) and the source, provide a link to the Creative Commons licence, and indicate if changes were made. The images or other third party material in this article are included in the article's Creative Commons licence, unless indicated otherwise in a credit line to the material. If material is not included in the article's Creative Commons licence and your intended use is not permitted by statutory regulation or exceeds the permitted use, you will need to obtain permission directly from the copyright holder. To view a copy of this licence, visit http://creativecommons.org/licenses/by/4.0/. 


\section{References}

1. Abdymanapov, S.A., Begehr, H., Tungatarov, A.B.: Some Schwarz problems in a quarter plane. Eurasian Math. J. 3, 22-35 (2005)

2. Abdymanapov, S.A., Begehr, H., Harutyunyan, G., Tungatarov, A.B.: Four boundary value problems for the Cauchy-Riemann equation in a quarter plane. In: More Progresses in Analysis, eds. H. Begehr et al., World Sci., Singapore, (2009), 1137-1147

3. Akel, M., Begehr, H.: Schwarz problem for first order elliptic systems in unbounded sectors. Eurasian Math. J. 5, 6-24 (2014)

4. Akel, M., Begehr, H.: Neumann function for a hyperbolic strip and a class of related plane domains. Math. Nachr. 290, 490-506 (2017). https://doi.org/10.1002/mana.201500501

5. Aksoy, Ü., Begehr, H., Çelebi, A.O.: Schwarz problem for higher-order complex partial differential equations in the upper half plane. Math. Nachr. 292, 1183-1193 (2019). https://doi.org/10.1002/mana. 201800028

6. Aksoy, Ü., Begehr, H., Çelebi, A.O.: A.V. Bitsadze's observation on bianalytic functions and the Schwarz problem. Complex Var. Elliptic Equ. 64(8), 1257-1274 (2019). https://doi.org/10.1080/ 17476933.2018.1504039

7. Aksoy, Ü., Begehr, H., Çelebi, A.O.: A.V. Bitsadze's observation on bianalytic functions and the Schwarz problem revisited. Complex Var. Elliptic Equ. 66(4), 583-585 (2021). https://doi.org/10. 1080/17476933.2020.1730825

8. Aksoy, Ü., Begehr, H., Çelebi, A.O., Shupeyeva, B.: Complex partial differential equations. Itogi Nauki i Tekhniki 188, 54-69 (2020). Russian

9. Aksoy, Ü., Çelebi, A.O.: Dirichlet problem for a generalized inhomogeneous polyharmonic equation in an annular domain. Complex Var. Elliptic Equ. 57(2-4), 229-241 (2012)

10. Begehr, H.: Complex analytic methods for partial differential equations. An introductory text. World Sci, Singapore (1994)

11. Begehr, H.: Boundary value problems in complex analysis, I, II. Bol. Asoc. Mat. Venezolana, XII (2005), 65-85, 217-250

12. Begehr, H.: Explicit solutions of the Poisson equation in plane domains. In: Modern Math. Methods High Performance Computing in Science and Technology, M3HPCST 2015, Ghaziabad, India, Dec. 2015, eds. V.K. Singh et al., Springer Proc. Math. and Statistics 171, Springer, New-Delhi, (2016), 111-127

13. Begehr, H.: Fundamental solutions to the Laplacian in plane domains bounded by ellipses. In: Mathematics and Computing, Third Intern. Conf., ICMC 2017, Haldia, India, Jan. 17-21, 2017, Proc., D. Giri, R.N. Mohapatra, H. Begehr, M.S. Obaidat (eds.). Comm. in Computer and Information Science 655, Springer, Singapore, (2017), 293-311

14. Begehr, H., Burgumbayeva, S., Shupeyeva, B.: Remark on Robin problem for Poisson equation. Complex Var. Elliptic Eqs. 62(10), 1589-1599 (2017). http://www.tandfonline.com/eprint/ W534YxAEU3RUBZDQ7Kqe/full; https://doi.org/10.1080/17476933.2017.1303052

15. Begehr, H., Burgumbayeva, S., Shupeyeva, B.: Harmonic Green functions for a plane domain with two touching circles as boundary. Adv. Math. Models Appl. 3, 18-29 (2018)

16. Begehr, H., Costache, M.-R., Tappert, S., Vaitekhovich, T.: Harmonic Green and Neumann representations in a triangle, quarter-disc and octo-plane. In: Progress in Analysis Appl., Proc. 7. Intern. ISAAC Cong., eds. M. Ruzhansky, J. Wirth. World Sci., Singapore, (2011), 74 - 80

17. Begehr, H., Hile, G.N.: A hierarchy of integral operators. Rocky Mountain J. Math. 27, 669-706 (1997)

18. Begehr, H., Kumar, A.: Boundary value problems for the inhomogeneous polyanalytic equation I. Analysis 25, 55-71 (2005)

19. Begehr, H., Schmersau, D.: The Schwarz problem for polyanalytic functions. ZAA 24, 341-351 (2005)

20. Begehr, H., Vaitekhovich, T.: How to find harmonic Green functions in the plane. Complex Var. Elliptic Eqs. 56, 1169-1181 (2011)

21. Begehr, H., Vaitekhovich, T.: The parqueting-reflection principle for constructing Green function. In: Analytic Methods of Analysis and Differential Equations: AMADE-2012 (edited by S.V. Rogosin, M.V. Dubatovskaya), Cambridge Sci. Publ., Cottenham, (2013), 11-20

22. Çelebi, A.O., Mityushev, V.V.: Schwarz problem for bi-analytic functions in multiply connected domains. Complex Var. Elliptic Equ. 60(4), 566-582 (2015)

23. Chaudhary, A., Kumar, A.: Boundary value problems in upper half plane. Complex Var. Ell. Eqs. 54, 441-448 (2009) 
24. Gaertner, E.: Basic complex boundary value problems in the upper half plane. Ph.D. thesis, FU Berlin, (2006); http://www.diss.fu-berlin.de/diss/receive/FUDISS_thesis_000000002129

25. Mityushev, V.V., Rogosin, S.V.: Constructive methods for linear and nonlinear boundary value problems for analytic functions: theory and applications. Chapman and Hall/CRC Monographs and Surveys in Pure and Applied Mathematics. 108. Boca Raton, FL: Chapman and Hall/CRC, (2000)

26. Shupeyeva, B.: Harmonic boundary value problems in a quarter ring domain. Adv. Pure Appl. Math. 3, 393-419 (2012)

27. Shupeyeva, B.: Some basic boundary value problems for complex partial differential equations in quarter ring and half hexagon. Ph.D. thesis, FU Berlin, (2013); http://www.diss.fu-berlin.de/diss/ receive/FUDISS_thesis_000000094596

28. Vaitsiakhovich, T.: Boundary value problems for complex partial differential equations in a ring domain. Ph.D. thesis, FU Berlin, (2008): http://www.diss.fu-berlin.de/diss/receive/FUDISS_thesis_ 000000003859

29. Vaitekhovich, T.S.: Boundary value problems to second order complex partial differential equations in a ring domain. Šiauliai Math. Seminar 2(10), 117-146 (2007)

30. Vaitekhovich, T.S.: Boundary value problems to first order complex partial differential equations in a ring domain. Integral Transf. Special Funct. 19, 211-233 (2008)

31. Vekua, I.N.: Generalized analytic functions. Transl. ed. by Ian N. Sneddon. International Series of Monographs on Pure and Applied Mathematics. Vol. 25. Oxford-London-New York-Paris: Pergamon Press 1962; Reading, Mass.- London: Addison-Wesley Publ. Co., Inc., (1962)

32. Wang, Ying: Boundary value problems for complex partial differetial equations in fan-shaped domains. Ph.D. thesis, FU Berlin, (2011); http://www.diss.fu-berlin.de/diss/receive/FUDISS_thesis_ 000000021359

33. Wang, Y.: Schwarz-type boundary value problems for the polyanalytic equation in the half unit disc. Complex Var. Elliptic Equ. 57(9), 983-993 (2012)

34. Wang, Y., Wang, Y.: On Schwarz-type boundary-value problems of polyanalytic equation on a triangle. Ann. Univ. Paedagog. Crac. Stud. Math. 9, 69-78 (2010)

Publisher's Note Springer Nature remains neutral with regard to jurisdictional claims in published maps and institutional affiliations. 